\title{
Experimental Investigation for Fault Diagnosis Based on a Hybrid Approach Using Wavelet Packet and Support Vector Classification
}

\author{
Pengfei Li, ${ }^{1,2}$ Yongying Jiang, ${ }^{1}$ and Jiawei Xiang ${ }^{1}$ \\ ${ }^{1}$ College of Mechanical and Electrical Engineering, Wenzhou University, Wenzhou 325035, China \\ ${ }^{2}$ School of Mechanical and Electrical Engineering, Guilin University of Electronic Technology, Guilin 541004, China
}

Correspondence should be addressed to Jiawei Xiang; wxw8627@163.com

Received 3 October 2013; Accepted 30 December 2013; Published 12 February 2014

Academic Editors: Y. Lei and M. Riera-Guasp

Copyright (C) 2014 Pengfei Li et al. This is an open access article distributed under the Creative Commons Attribution License, which permits unrestricted use, distribution, and reproduction in any medium, provided the original work is properly cited.

\begin{abstract}
To deal with the difficulty to obtain a large number of fault samples under the practical condition for mechanical fault diagnosis, a hybrid method that combined wavelet packet decomposition and support vector classification (SVC) is proposed. The wavelet packet is employed to decompose the vibration signal to obtain the energy ratio in each frequency band. Taking energy ratios as feature vectors, the pattern recognition results are obtained by the SVC. The rolling bearing and gear fault diagnostic results of the typical experimental platform show that the present approach is robust to noise and has higher classification accuracy and, thus, provides a better way to diagnose mechanical faults under the condition of small fault samples.
\end{abstract}

\section{Introduction}

The bearing and gear are the most critical and frequently encountered components in vast majority of rotating machinery. Their operating state directly affects the machine performance, efficiency, and life. Therefore, fault identification of rolling element bearing and gear has been the subject of extensive research.

Vibration analysis has been established as the most common and reliable method of analysis. Generally, the vibration signals can be used to detect the incipient fault of the machine components and reduce the possibility of catastrophic damage and the down time, through the on-line monitoring and diagnosis system $[1,2]$. The extracted features include time domain features such as root mean square, variance, skewness, and kurtosis [3-5], frequency domain features such as content at the feature frequency and the amplitudes of frequency spectrum [6,7], and time frequency domain features such as the statistical characteristics of shorttime Fourier transform (STFT), Wigner-Viller distribution (WVD), wavelet transform (WT), and so forth [8-10]. The WT method possesses perfect local property in both time space and frequency space, and it is used widely in the region of machinery fault detection and identification [11-13]. However, the WT cannot split the high frequency band where the modulation information of machine fault is often involved in. The wavelet package transform (WPT) can overcome the difficulty. Nikolaou and Antoniadis proposed a method for the analysis of vibration signals resulting from bearing with localized defects using the wavelet packet transform [14]. Fan and Zuo combined Hilbert transform and wavelet packet transforms to extract modulating signal and detect the early gear fault [15]. Wang and Lin investigated fault signals denoising processing using wavelet packet decomposition coefficients to identify the weak fault characteristic frequency of rolling bearings under strong background noise [16] However, these investigations did not combine intelligent fault diagnosis techniques to further recognize faults. The carefully selected vibration signals are necessary to match the theoretical fault frequency. For this reason, the advantages of WPT are not reflected.

Many intelligent classification algorithms, such as artificial neural networks (ANNs) and support vector classification (SVC), have been proposed to detect mechanical faults and recognize machine conditions [1-3]. The main difference between ANNs and SVC is in their risk minimization. In the 


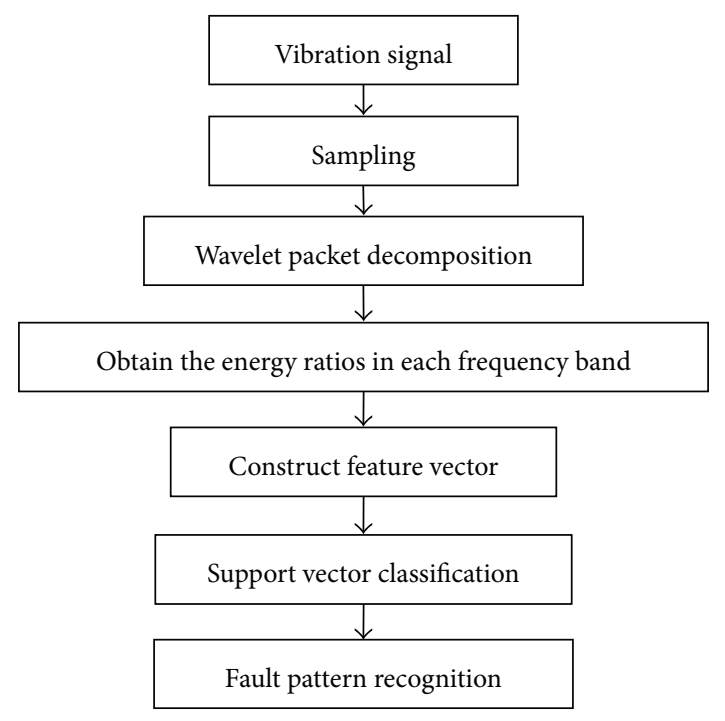

FIGURE 1: Fault pattern recognition flowchart.

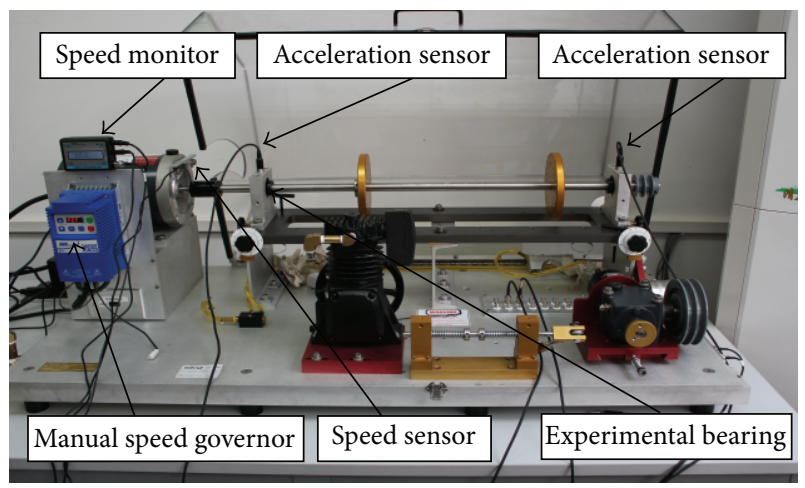

FIGURE 2: The MFS-MG experimental platform.

case of SVC, structural risk minimization principle is used to minimize an upper bound based on an expected risk. In ANNs, traditional empirical risk minimization is employed to minimize the error in training of data. The difference in risk minimization leads to a better generalization performance for SVC than ANNs. Thukaram et al. [17] compared the differences between the ANNs and SVC in identifying the fault. Crampton and Mason [18] found that when the data contains noise, the fault detection using support vector machine (SVM) is more effective than other intelligent techniques. However, only ANNs or SVC can not obtain satisfactory classification results from high level ambient noise. Therefore, in recent years, more and more researchers focus on the hybrid approach using WPT and SVC for fault classification. Bin et al. [19] combined WPT and empirical mode decomposition to extract fault feature frequency and further employed ANNs to detect faults in rotating machinery. $\mathrm{Hu}$ et al. [20] presented a hybrid approach for bearing fault diagnosis using WPT and SVC. Xian and Zeng developed Hu's scheme for bearing fault diagnosis using WPT and hybrid SVC [21]. Shen et al. proposed a new scheme using the extraction of statistical parameters from
WPT of original signals, a distance evaluation technique, and a support vector regression (SVR) based generic multiclass solver [22]. However, due to the limitation of machinery fault simulator, the fault samples used in the above investigations came from a single data source, mostly from the Case Western Reserve University official website. Therefore, the superiority of the hybrid approaches is not confirmed.

For the above reasons, this paper presents a hybrid approach for bearing and gear fault diagnosis based on a hybrid approach using wavelet packet decomposition and SVC. To validate the proposed method, we carry out experimental investigations using the machinery fault simulator (MFS-MG). A large number of experimental data is collected for bearing and gear under different working conditions. Our test results have shown that the proposed approach is effective and can further detect mechanical faults with an agreeable precision.

\section{Wavelet Packet Decomposition and Support Vector Classification Diagnostic Principles}

The fault pattern recognition flowchart based on wavelet packet decomposition and SVC is shown in Figure 1. The wavelet packet is employed to decompose the vibration signal to obtain the energy ratio in each frequency band. Taking these energy ratios as feature vectors, we can detect faults from the determined fault type through the trained SVC. The detailed procedures are described in Sections 2.1 and 2.2.

\subsection{Wavelet Packet Decomposition to Extract Feature Vector}

2.1.1. 3-Layer Decomposition for Each Signal Using Wavelet Packet. When decomposing the vibration signal using wavelet packet, the binary tree structure will be obtained. The final layer of the binary tree structure of the energy ratio in each frequency band can be obtained through calling the wenergy function. According to WPT theory, index $(i, j)$ represents the $i$ th layer and the $j$ th node $\left(j=2^{i}\right)$ as well as a certain signal component (frequency band). For example, $(0,1)$ represents the original signal, $(1,1)$ represents the low frequency wavelet packet decomposition coefficients of the first layer, and $(1,2)$ represents the high frequency wavelet packet decomposition coefficients of the same layer. For the 3-layer $(i=3)$ case, the corresponding node $j=1,2, \ldots, 8$. Therefore, we have eight indexes $(3, j)$ representing eight signal components (frequency bands).

2.1.2. Obtaining the Energy Ratio in Each Frequency Band. Generally, the frequency bands are not arranged in accordance with the frequency order from low to high. When the original vibration signal is decomposed by wavelet packet (through the high-pass filter and downsampling procedure), the spectral sequence will be flipped. Therefore, to calculate the energy ratios, we should adjust the order of the corresponding frequency bands. For the 3-layer $(i=3)$ case, the exact frequency bands in accordance with the frequency from 


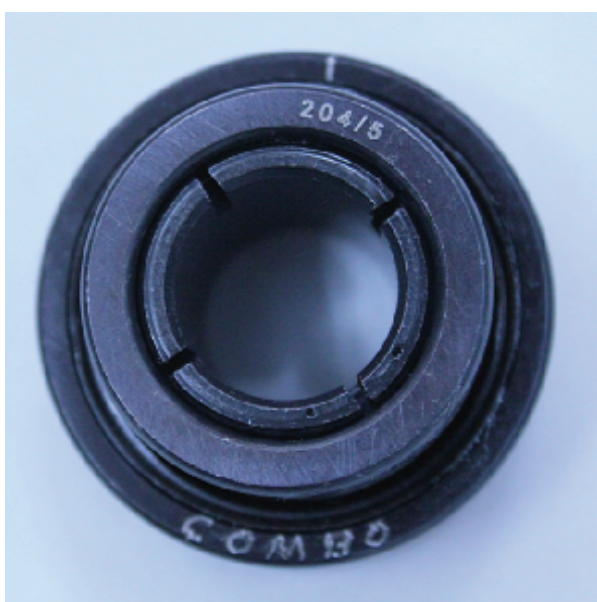

(a) A bearing with compound faults

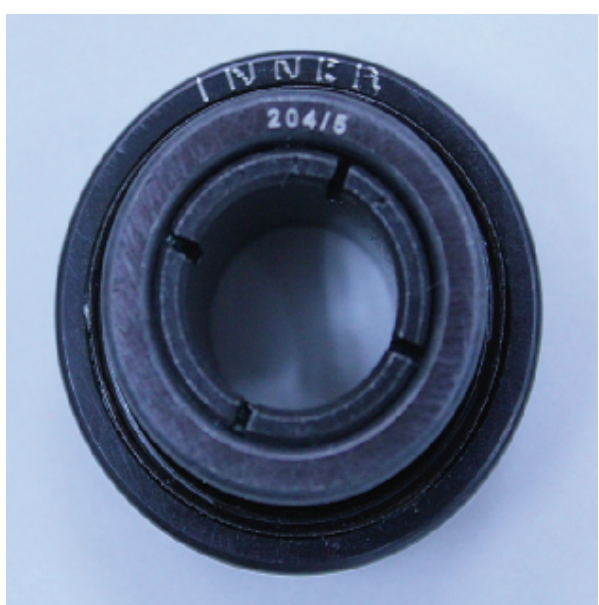

(c) A bearing with inner race fault

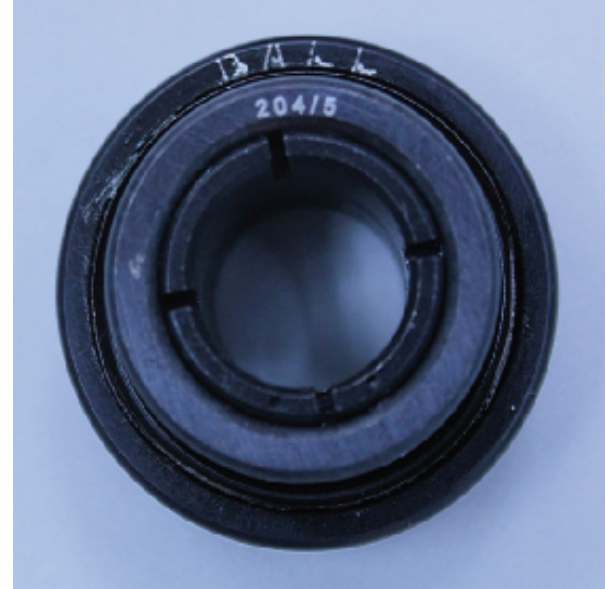

(b) A bearing with rolling element fault

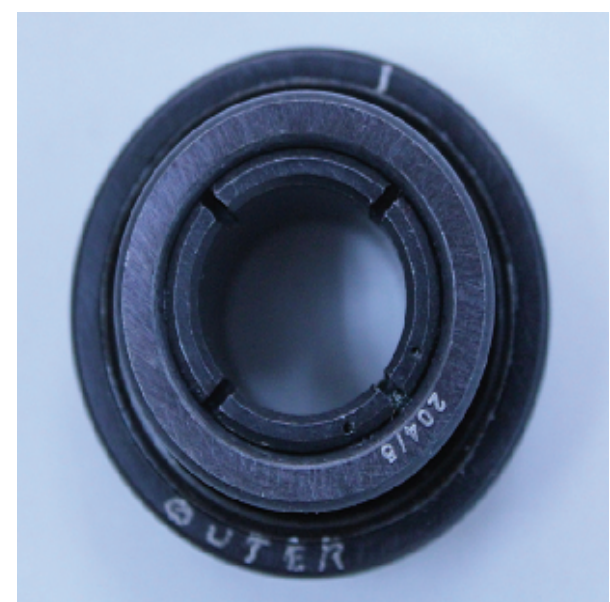

(d) A bearing with outer race fault

FIGURE 3: Bearings with typical faults.
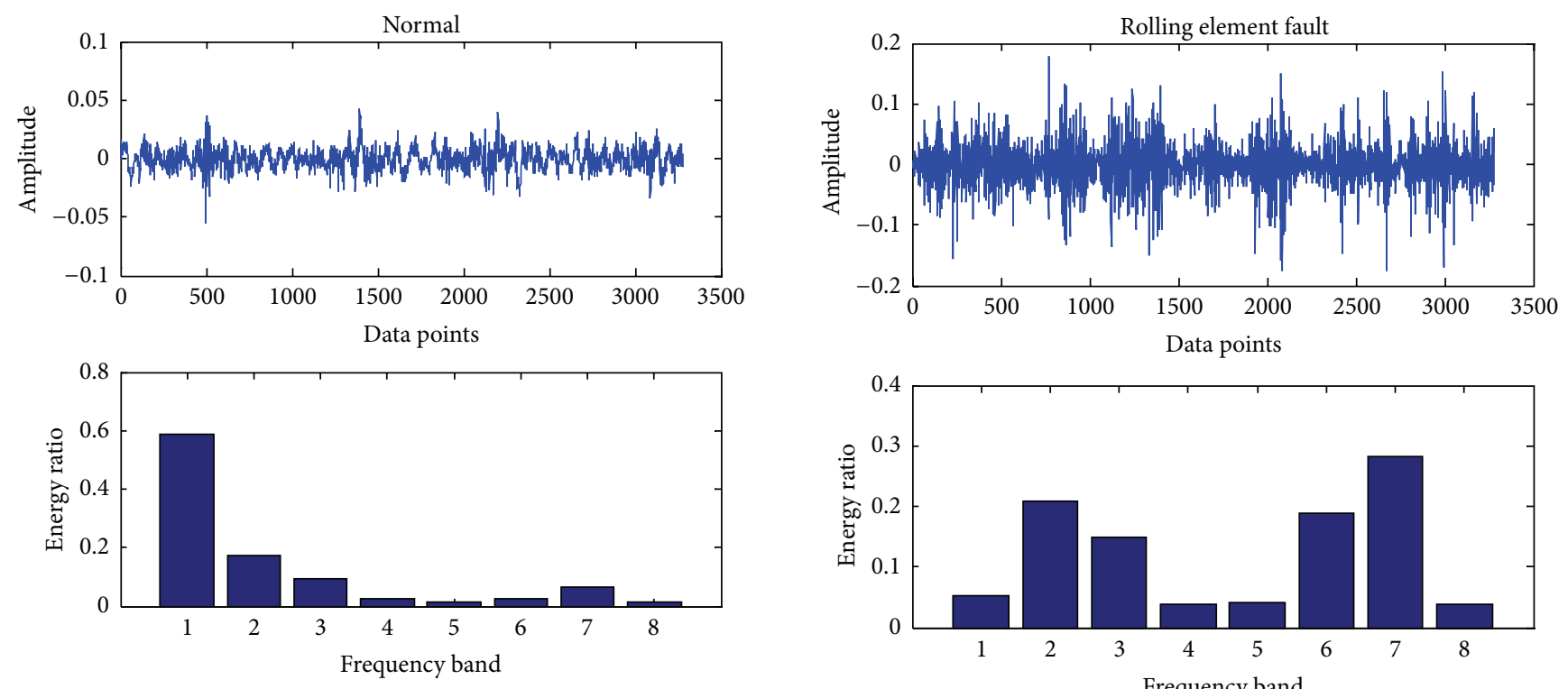

FIGURE 4: Normal case of a bearing.

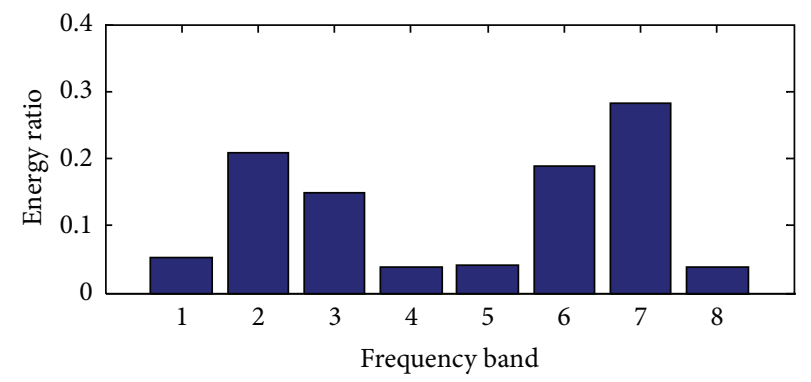

FIgURE 5: Rolling element fault. 

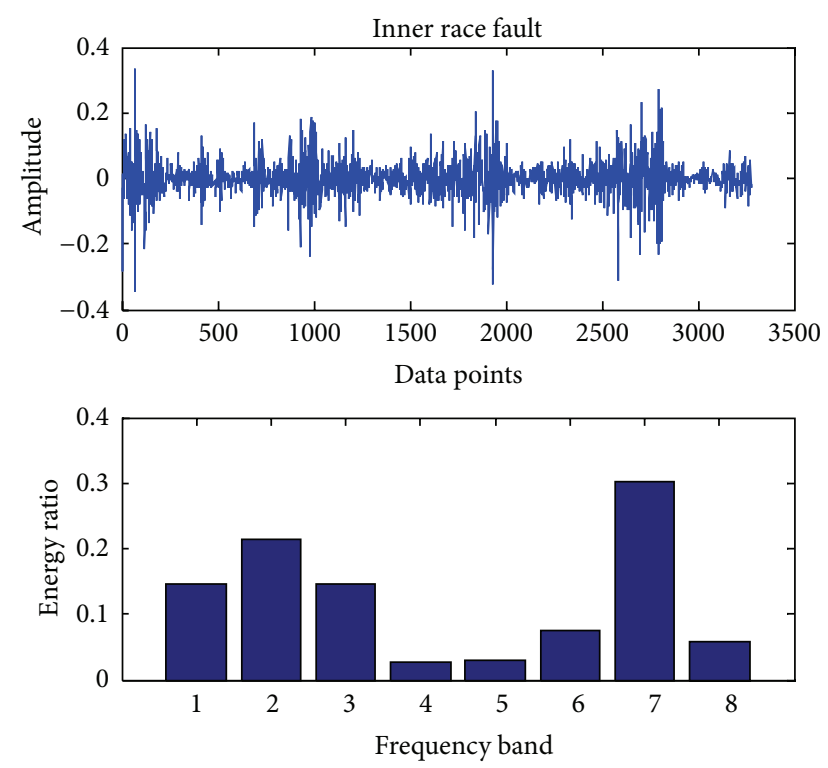

FIGURE 6: Inner race fault.
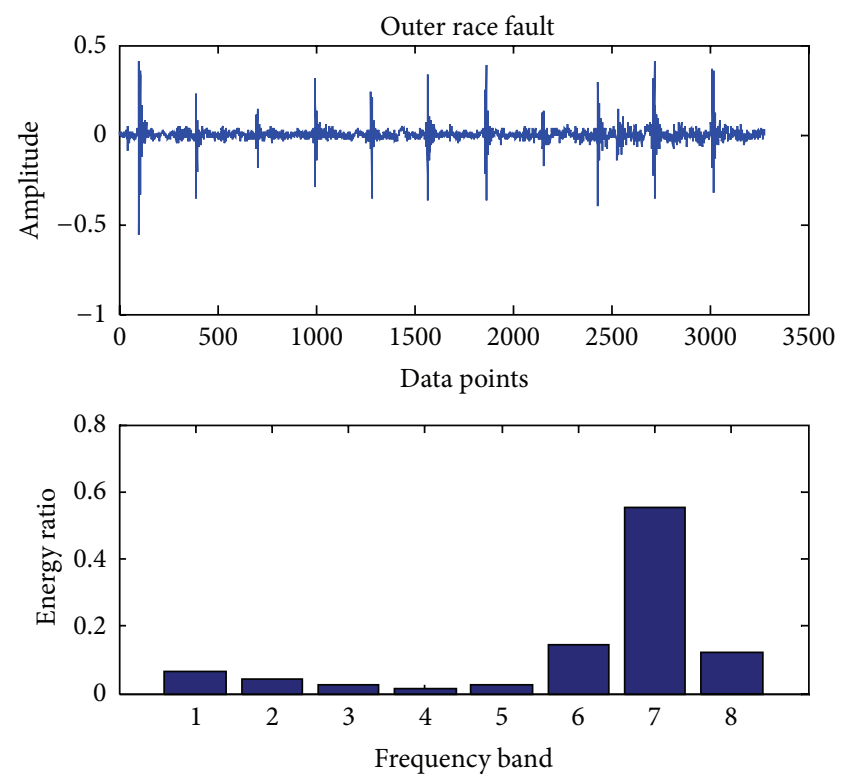

Figure 7: Outer race fault.

low to high should be $(3,1),(3,2),(3,4),(3,3),(3,7),(3,8)$, $(3,6)$, and $(3,5)[23]$.

2.1.3. Construct Feature Vector. The energy of the vibration component in each band will be changed when the faults of the mechanical system occurred. For the different faults, the energy ratios will be changed accordingly. Therefore, for the 3-layer $(i=3)$ case of wavelet packet decomposition, a feature vector $\mathbf{T}$ can be constructed by the eight energy ratios as

$$
\mathbf{T}=\left\{E_{31}, E_{32}, E_{33}, E_{34}, E_{35}, E_{36}, E_{37}, E_{38}\right\} .
$$

Here, $E_{3 j}(j=1,2,3, \ldots, 8)$ represent all of the energy ratios for the 3 rd layer and the $j$ th node.
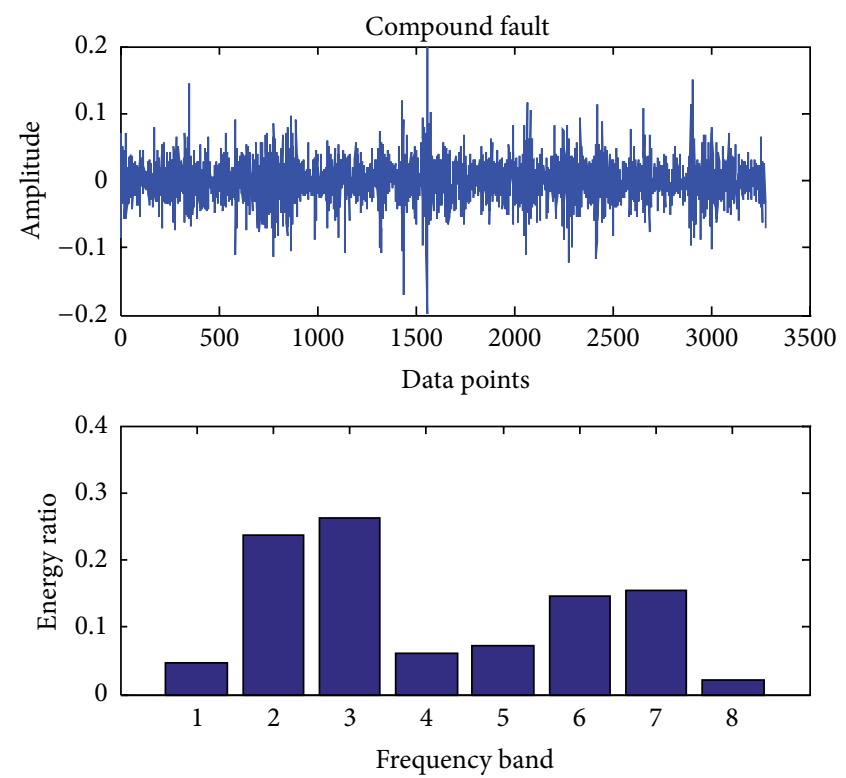

FIgURE 8: Compound fault of a bearing.

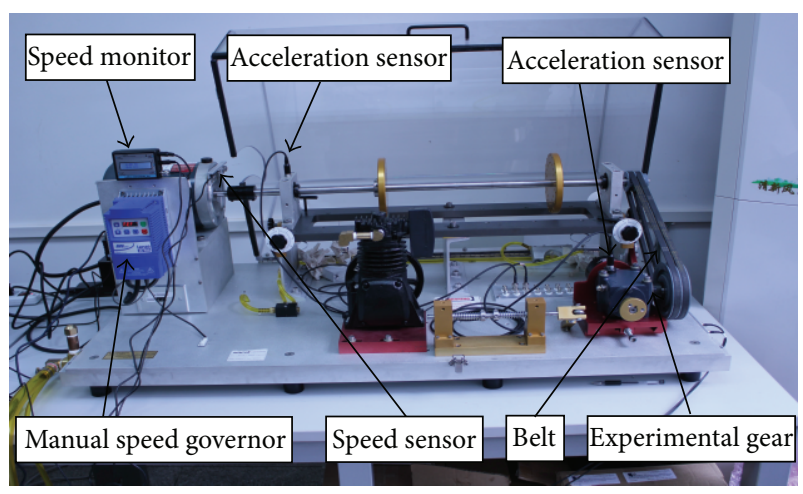

FIGURE 9: The MFS-MG experimental platform.

2.2. Fault Pattern Recognition Using SVC. The basic theory for SVC is summarized in this section [24].

Assume that a training set $S$ is given by

$$
S=\left\{x_{i}, y_{i}\right\}_{i=1}^{n},
$$

where $x_{i} \in R^{n}$ and $y_{i} \in\{-1,+1\}$. The goal of SVMs is to find an optimal hyper plane such that

$$
\begin{aligned}
& w^{T} x_{i}+b \geq 1, \quad \text { for } y_{i}=+1, \\
& w^{T} x_{i}+b \leq 1, \quad \text { for } y_{i}=-1,
\end{aligned}
$$

where the weight vector $w \in R^{n}$ and the bias $b$ is a scalar.

If the inequality in (3) holds for all training data, it will be a linearity separable case. Therefore, to find the optimal hyper plane, one can solve the following constrained optimization problem:

$$
\begin{array}{ll}
\text { Minimize } & \Phi(w)=\frac{1}{2} w^{T} w \\
\text { Subject to } & y_{i}\left(w^{T} x_{i}+b\right) \geq 1, \quad i=1,2, \ldots, n .
\end{array}
$$




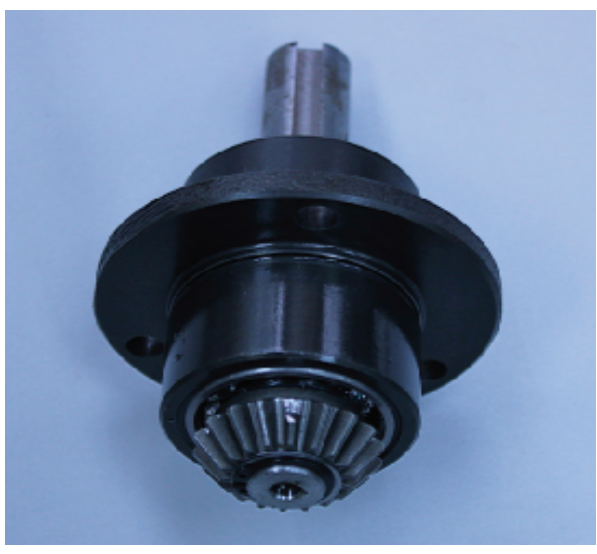

(a) A gear with broken teeth

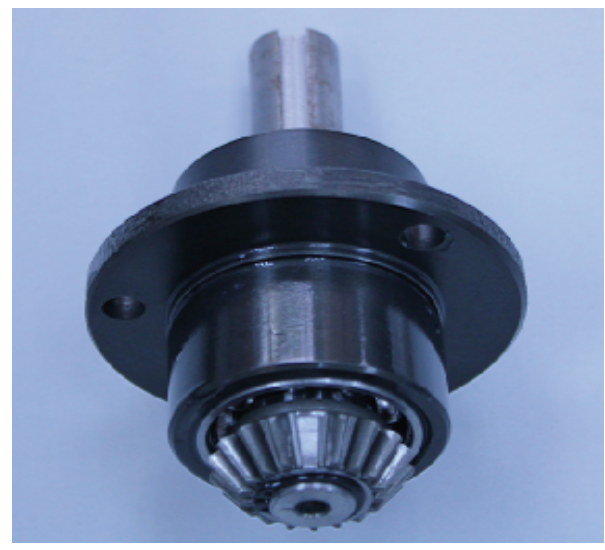

(b) A gear with missing teeth

FIgURE 10: Typical fault gear.
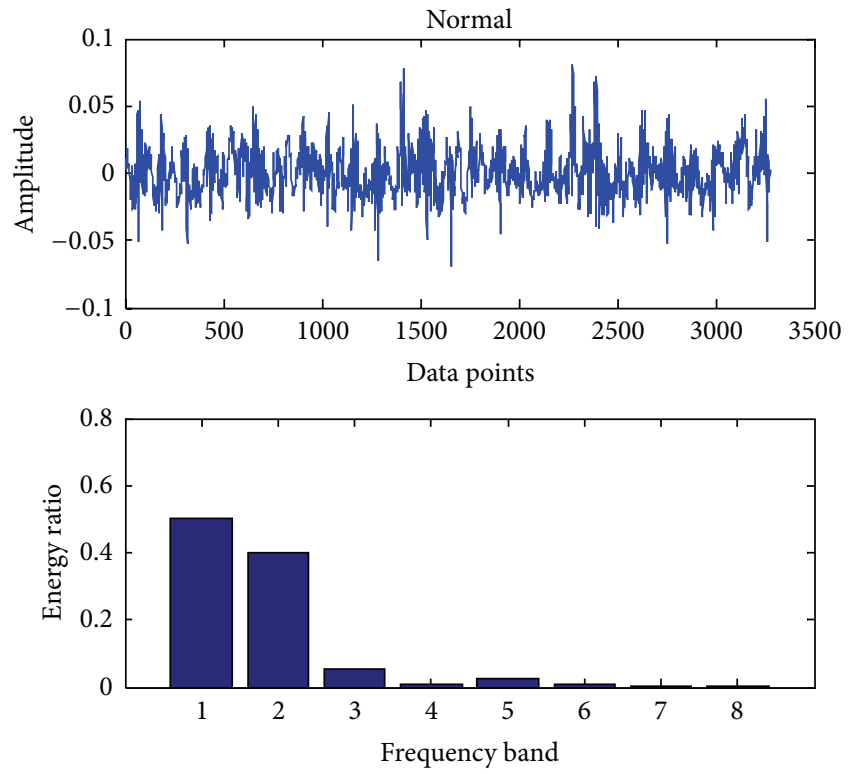

FIgURE 11: Normal case of a gear.

If inequality in (3) does not hold for some data points in $S$, SVMs become linearly not separable. To find an optimal hyper plane, we have to solve the following constrained optimization problem:

$$
\begin{array}{cl}
\text { Minimize } & \Phi(w)=\frac{1}{2} w^{T} w+C \sum_{i=1}^{n} \xi_{i} \\
\text { Subject to } & y_{i}\left(w^{T} x_{i}+b\right) \geq 1-\xi_{i}, \\
& \xi_{i} \geq 0, \quad i=1,2, \ldots, n .
\end{array}
$$

By introducing a set of Lagrange multipliers $\alpha_{i}, \beta_{i}$ for constraints, the problem becomes the one to find the saddle point of the Lagrangian. Therefore, the dual problem becomes
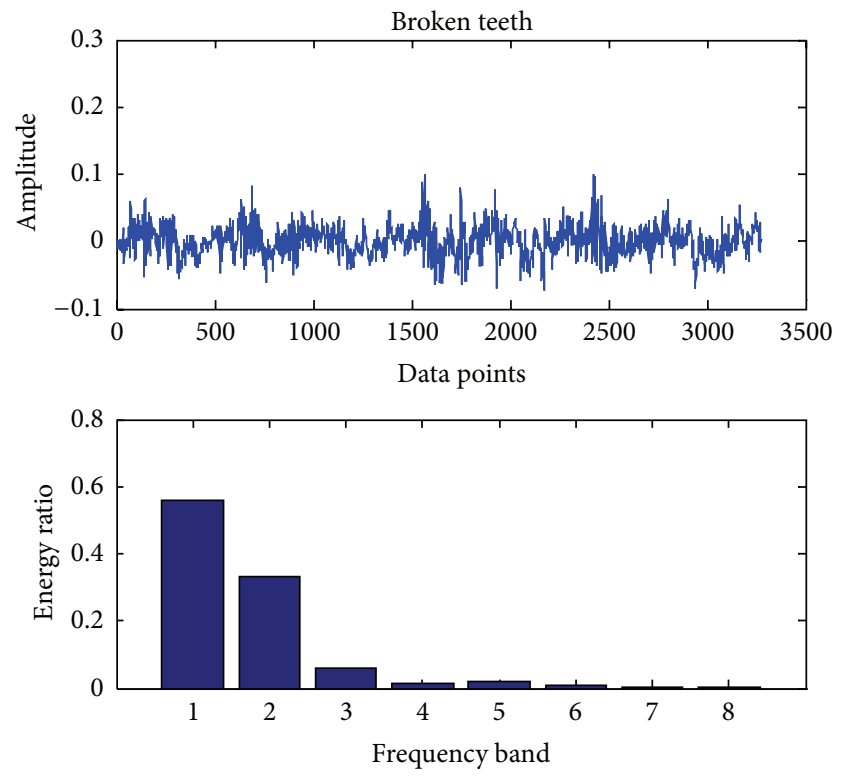

FIGURE 12: Gear broken teeth.

$$
\begin{array}{ll}
\text { Minimize } & Q(\alpha)=\sum_{i=1}^{n} \alpha_{i}-\frac{1}{2} \sum_{i=1}^{n} \sum_{j=1}^{n} \alpha_{i} \alpha_{j} y_{i} y_{j} x_{i}^{T} x_{j} \\
\text { Subject to } & \sum_{i=1}^{n} \alpha_{i} y_{i}=0, \\
& 0 \leq \alpha_{i} \leq C, \quad i=1,2, \ldots, n .
\end{array}
$$

If $0<\alpha_{i} \leq C$, the corresponding data points are called support vectors (SVs).

SVMs map the input vector into a higher dimensional feature and, thus, can solve the nonlinear case. By choosing a nonlinear mapping function $\varphi(x) \in R^{m}$, where $M>N$, the SVM can construct an optimal hyper plane in the new 

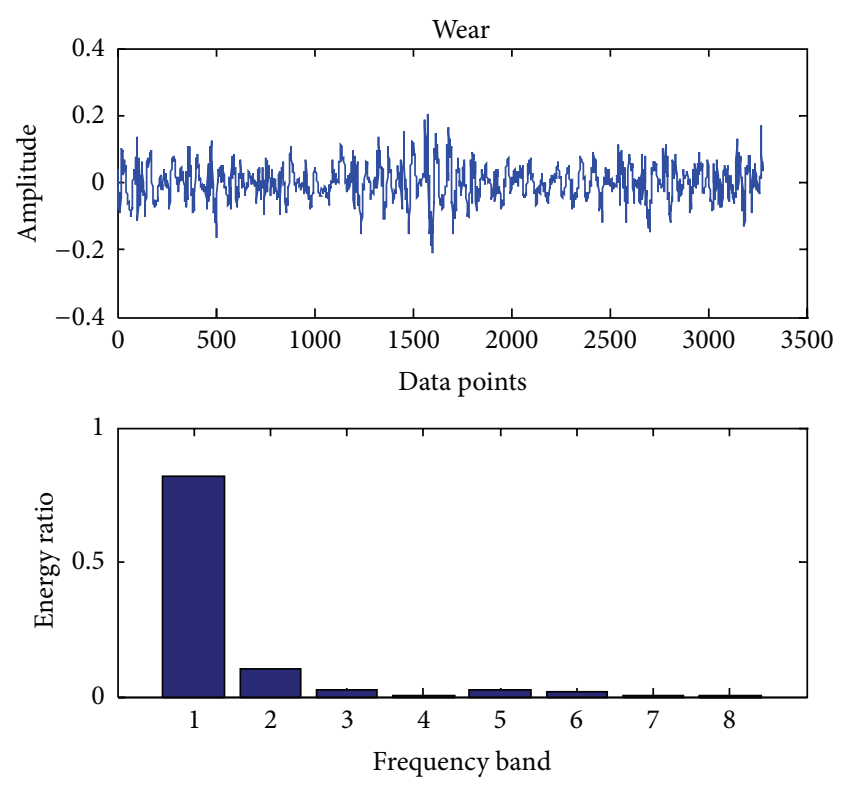

FIGURE 13: Gear wear.
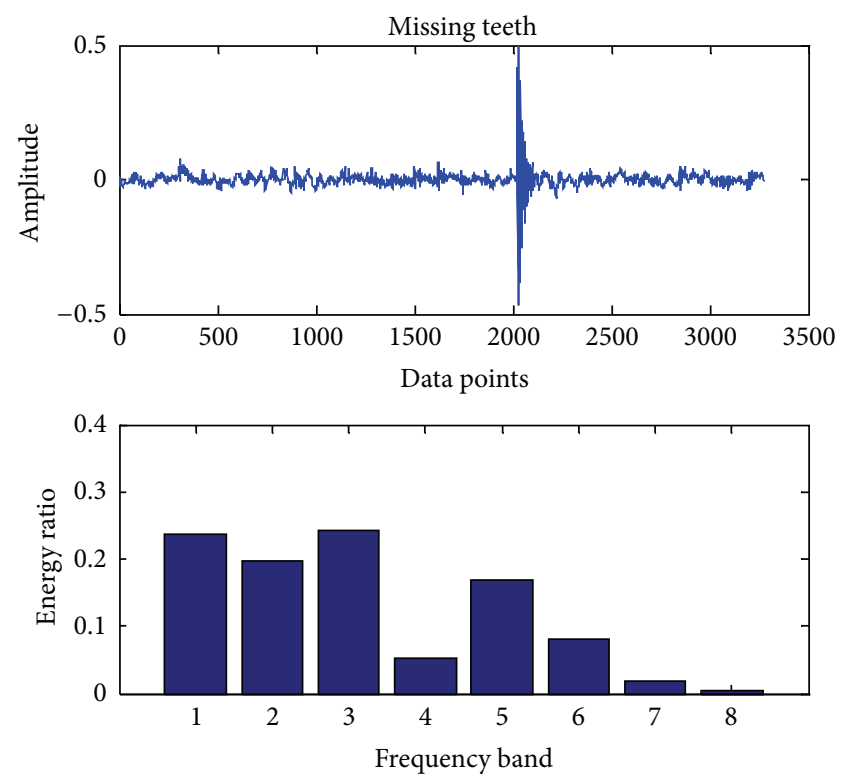

FIGURE 14: Gear missing teeth.

feature space. $K\left(x, x_{i}\right)$ is the inner product kernel performing the nonlinear mapping into feature space as

$$
K\left(x, x_{i}\right)=K\left(x_{i}, x\right)=\varphi(x)^{T} \varphi\left(x_{i}\right) .
$$

Therefore, the dual optimization problem becomes

$$
\text { Minimize } \quad Q(\alpha)=\sum_{i=1}^{n} \alpha_{i}-\frac{1}{2} \sum_{i=1}^{n} \sum_{j=1}^{n} \alpha_{i} \alpha_{j} y_{i} y_{j} K\left(x_{i}, x_{j}\right)
$$

and the constraints are the same as shown in (7) and (8); the only requirement on the kernel $K\left(x, x_{i}\right)$ is to satisfy the
Mercer's theorem [24]. Using Kernel functions, every data will be classified as

$$
x \in \begin{cases}\text { positive class, } & \text { if } g(x)>0 \\ \text { negative class, } & \text { if } g(x)<0\end{cases}
$$

in which the decision function is

$$
g\left(x_{i}\right)=y_{i}\left(\sum_{j=1}^{N} y_{j} \alpha_{j} K\left(x_{i}, x_{j}\right)+b\right) .
$$

The typical examples of kernel function are polynomial kernel, radial basis function (RBF) kernel, sigmoid kernel, and linear kernel. In many practical applications [25-27], the RBF kernel obtains the highest classification accuracy rate than other kernel functions. Therefore, the RBF kernel is employed in the present investigation.

Support vector machines were originally designed for binary classification. How to effectively extend it for multiclass classification is still an ongoing research issue. Currently there are several methods that have been proposed for multiclass classification, such as one-against-one, one-against-all, and directed acyclic graph (DAG). Hsu and Lin [28] gave a comparison of these methods and pointed out that the oneagainst-one method is more suitable for practical use than others. In the present, the one-against-one method is applied to detect the faults of bearings and gears.

\section{Experimental Investigation}

In this section, two typical fault diagnosis experiments for bearings and gears are given to testify the performance of the proposed hybrid approach.

3.1. Bearing Fault Diagnosis. The MFS-MG experimental platform for the bearing fault simulation [29] is shown in Figure 2. It includes speed monitor, manual speed governor, acceleration sensors, speed sensor, motor, spindle, bearings, and so forth. During the experiment, the data are acquired by an accelerometer mounted on the top of the bearing holder on left side. The vibration signals of the five fault types are collected, that is, normal case, rolling element fault, inner race fault, outer race fault, and compound fault (including inner race, outer race, and rolling element faults). The spindle speed is $1792 \mathrm{rpm}$ and the end of the experimental bearing (see Figure 2) is free of loading. The bearings with typical faults are shown in Figure 3: the bearing model is ER-12K, the bearing pitch diameter is $33.4772 \mathrm{~mm}$, the number of rolling element is 8, and the rolling element diameter is $7.9375 \mathrm{~mm}$. Figures 3(a), 3(b), 3(c), and 3(d) show four fault cases, that is, a bearing with compound faults, a bearing with rolling element fault, a bearing with inner race fault, and a bearing with outer race fault, respectively.

In the present, the sampling frequency is $25.6 \mathrm{k} .163800$ data points (bearing vibration signal) are collected at one running in different cases and divided into 50 sections. Each signal contains 3276 data points. Db18 wavelet [23] is used to decompose each signal into three layers and gain 8 subbands in the final layer. The energy ratio of each band 
TABLE 1: Bearing training samples.

\begin{tabular}{|c|c|c|c|c|c|c|c|c|}
\hline \multirow{2}{*}{ Cases } & \multicolumn{8}{|c|}{ Energy ratios in each frequency band } \\
\hline & 1 & 2 & 3 & 4 & 5 & 6 & 7 & 8 \\
\hline \multirow{3}{*}{ Normal } & 0.5862 & 0.1738 & 0.0930 & 0.0252 & 0.0165 & 0.0263 & 0.0638 & 0.0155 \\
\hline & 0.4725 & 0.2200 & 0.1097 & 0.0295 & 0.0253 & 0.0338 & 0.0899 & 0.0193 \\
\hline & 0.5887 & 0.1845 & 0.0815 & 0.0210 & 0.0148 & 0.0219 & 0.0707 & 0.0168 \\
\hline \multirow{3}{*}{ Rolling element fault } & 0.0519 & 0.2047 & 0.1484 & 0.0389 & 0.0457 & 0.1648 & 0.3067 & 0.0390 \\
\hline & 0.0556 & 0.1537 & 0.1239 & 0.0471 & 0.0544 & 0.1664 & 0.3229 & 0.0761 \\
\hline & 0.0516 & 0.1457 & 0.0830 & 0.0415 & 0.0533 & 0.1692 & 0.4006 & 0.0551 \\
\hline \multirow{3}{*}{ Inner race fault } & 0.1458 & 0.2153 & 0.1452 & 0.0272 & 0.0292 & 0.0757 & 0.3030 & 0.0586 \\
\hline & 0.1691 & 0.2262 & 0.1234 & 0.0375 & 0.0446 & 0.0780 & 0.2779 & 0.0433 \\
\hline & 0.0857 & 0.1596 & 0.1210 & 0.0238 & 0.0343 & 0.1040 & 0.3967 & 0.0749 \\
\hline \multirow{3}{*}{ Outer race fault } & 0.0664 & 0.0448 & 0.0259 & 0.0129 & 0.0277 & 0.1449 & 0.5550 & 0.1224 \\
\hline & 0.0636 & 0.0410 & 0.0165 & 0.0111 & 0.0215 & 0.1190 & 0.5888 & 0.1386 \\
\hline & 0.0740 & 0.0311 & 0.0141 & 0.0090 & 0.0249 & 0.1745 & 0.5404 & 0.1321 \\
\hline \multirow{3}{*}{ Compound fault } & 0.0457 & 0.2360 & 0.2616 & 0.0623 & 0.0731 & 0.1457 & 0.1544 & 0.0213 \\
\hline & 0.0464 & 0.1528 & 0.1851 & 0.0691 & 0.0695 & 0.1925 & 0.2556 & 0.0291 \\
\hline & 0.0553 & 0.1896 & 0.2769 & 0.0748 & 0.0770 & 0.1263 & 0.1774 & 0.0228 \\
\hline
\end{tabular}

TABLE 2: Bearing test samples.

\begin{tabular}{|c|c|c|c|c|c|c|c|c|}
\hline \multirow{2}{*}{ Cases } & \multicolumn{8}{|c|}{ Energy ratios in each frequency band } \\
\hline & 1 & 2 & 3 & 4 & 5 & 6 & 7 & 8 \\
\hline \multirow{3}{*}{ Normal } & 0.5318 & 0.2206 & 0.0832 & 0.0256 & 0.0198 & 0.0292 & 0.0718 & 0.0180 \\
\hline & 0.5826 & 0.1843 & 0.0945 & 0.0200 & 0.0178 & 0.0232 & 0.0628 & 0.0148 \\
\hline & 0.5623 & 0.1877 & 0.0938 & 0.0209 & 0.0221 & 0.0251 & 0.0722 & 0.0160 \\
\hline \multirow{3}{*}{ Rolling element fault } & 0.0553 & 0.1541 & 0.1091 & 0.0403 & 0.0624 & 0.1990 & 0.3271 & 0.0528 \\
\hline & 0.1066 & 0.2799 & 0.0772 & 0.0219 & 0.0339 & 0.1315 & 0.3022 & 0.0469 \\
\hline & 0.0364 & 0.0993 & 0.0677 & 0.0355 & 0.0365 & 0.1707 & 0.4851 & 0.0686 \\
\hline \multirow{3}{*}{ Inner race fault } & 0.1295 & 0.1626 & 0.1031 & 0.0294 & 0.0504 & 0.0944 & 0.3612 & 0.0693 \\
\hline & 0.1548 & 0.2436 & 0.1546 & 0.0434 & 0.0392 & 0.0749 & 0.2385 & 0.0511 \\
\hline & 0.1403 & 0.1962 & 0.1596 & 0.0286 & 0.0309 & 0.0794 & 0.3197 & 0.0452 \\
\hline \multirow{3}{*}{ Outer race fault } & 0.0428 & 0.0640 & 0.0319 & 0.0170 & 0.0190 & 0.1508 & 0.5503 & 0.1243 \\
\hline & 0.0304 & 0.0416 & 0.0238 & 0.0117 & 0.0230 & 0.1421 & 0.5469 & 0.1805 \\
\hline & 0.0598 & 0.0867 & 0.0330 & 0.0135 & 0.0196 & 0.1422 & 0.5166 & 0.1285 \\
\hline \multirow{3}{*}{ Compound fault } & 0.0388 & 0.1612 & 0.1681 & 0.0670 & 0.0679 & 0.2692 & 0.1964 & 0.0313 \\
\hline & 0.0533 & 0.1979 & 0.2272 & 0.0811 & 0.0737 & 0.1538 & 0.1905 & 0.0224 \\
\hline & 0.0636 & 0.2239 & 0.1841 & 0.0691 & 0.0629 & 0.1777 & 0.1941 & 0.0246 \\
\hline
\end{tabular}

is obtained through calling the wenergy function in the wavelet toolbox of Matlab. Therefore, as shown in (1), a $1 \times$ 8 vector (feature vector) is obtained. Figures $4,5,6,7$, and 8 , respectively, represent the graph of the original signals and the corresponding eight energy ratios distribution maps for five cases including normal case and four fault cases.

For each of the five cases, we collect two bearing vibration signals (each signal contains 163800 data points) at two runnings. The first signal is employed to train the SVM and the second signal to be tested. According to the above description, 50 feature vectors can be extracted from each signal. Therefore, 50 feature vectors (samples) in the first running are served as training samples and the other 50 feature vectors (samples) in the second running are the fault samples to be tested (classified). Tables 1 and 2 give the first three training samples of the first signal and the first three test samples of the second signal. To represent the five cases numerically, we label the normal case, the rolling element fault, the inner race fault, the outer race fault, and the compound fault as 1 to 5 , respectively. It points out that they are called standard labels.

In the present investigation, we adopt the SVM toolkit programmed by Franc and Hlavác of the Czech Technical University [30].

For general analysis, it is desirable to use normalized, nondimensional parameters. The normalized parameters also speed up the computational process. Therefore, prior to the training of the SVC model, all samples data are normalized to be bounded by $[0,1]$. 
TABLE 3: Bearing recognition results.

\begin{tabular}{lcccc}
\hline Cases & Training samples & Testing samples & Classification label & Recognition rate \\
\hline Normal & 50 & 50 & 1 & 2 \\
Rolling element fault & 50 & 50 & 3 & $86 \%$ \\
Inner race fault & 50 & 50 & 4 & $96 \%$ \\
Outer race fault & 50 & 50 & 5 & $100 \%$ \\
Compound fault & 50 & 50 & & $100 \%$ \\
\hline
\end{tabular}

TABLE 4: Gear training samples.

\begin{tabular}{|c|c|c|c|c|c|c|c|c|}
\hline \multirow{2}{*}{ Cases } & \multicolumn{8}{|c|}{ Energy ratios in each frequency band } \\
\hline & 1 & 2 & 3 & 4 & 5 & 6 & 7 & 8 \\
\hline \multirow{3}{*}{ Normal } & 0.5022 & 0.3977 & 0.0530 & 0.0077 & 0.0250 & 0.0098 & 0.0034 & 0.0012 \\
\hline & 0.4511 & 0.4109 & 0.0716 & 0.0079 & 0.0407 & 0.0128 & 0.0038 & 0.0013 \\
\hline & 0.4911 & 0.3799 & 0.0651 & 0.0080 & 0.0360 & 0.0150 & 0.0037 & 0.0011 \\
\hline \multirow{3}{*}{ Broken teeth } & 0.5591 & 0.3319 & 0.0615 & 0.0152 & 0.0224 & 0.0065 & 0.0029 & 0.0007 \\
\hline & 0.6000 & 0.2979 & 0.0578 & 0.0130 & 0.0226 & 0.0060 & 0.0023 & 0.0005 \\
\hline & 0.5589 & 0.3253 & 0.0686 & 0.0146 & 0.0216 & 0.0073 & 0.0031 & 0.0006 \\
\hline \multirow{3}{*}{ Wear } & 0.8194 & 0.1025 & 0.0226 & 0.0059 & 0.0266 & 0.0206 & 0.0018 & 0.0006 \\
\hline & 0.8289 & 0.0902 & 0.0178 & 0.0056 & 0.0380 & 0.0173 & 0.0016 & 0.0005 \\
\hline & 0.8080 & 0.1084 & 0.0209 & 0.0061 & 0.0391 & 0.0155 & 0.0018 & 0.0003 \\
\hline \multirow{3}{*}{ Missing teeth } & 0.2358 & 0.1971 & 0.2427 & 0.0528 & 0.1688 & 0.0814 & 0.0177 & 0.0035 \\
\hline & 0.1239 & 0.2404 & 0.1706 & 0.0746 & 0.2052 & 0.1422 & 0.0387 & 0.0044 \\
\hline & 0.2064 & 0.3353 & 0.1174 & 0.0537 & 0.1634 & 0.1051 & 0.0141 & 0.0046 \\
\hline
\end{tabular}

According to the SVC algorithm, the samples and label are

$$
\begin{aligned}
& \left\{\mathbf{X}_{i}, H_{i}^{s}\right\}_{i=1}^{l}, \\
& \mathbf{X}_{i}=\left\{E_{31}, E_{32}, E_{33}, E_{34}, E_{35}, E_{36}, E_{37}, E_{38}\right\}, \\
& \text { for } H_{i}^{s}=y
\end{aligned}
$$

where $\mathbf{X}_{i}$ and $H_{i}^{s}$ are, respectively, the samples and label and $l$ is the number of samples. From the above, the data for samples constitute a $50 \times 8$ matrix and the label lead to a $50 \times 1$ vector. Then, the first signal (50 segments) in all cases inputs the support vector machine for training. That means it includes two matrices, that is, the $250 \times 8$ matrix of samples and the $250 \times 1$ vector of label. After training, the second signal (50 segments) in all cases separately inputs the trained SVM for testing. Therefore, it includes a $50 \times 8$ vector of test samples. The predicted output (predicted label) will be obtained when the test is completed, and the recognition rate of every case can be obtained through the comparison of the predicted label with the standard labels. For each training and prediction, $\arg , C(\arg =1, C=10$, suggested by Franc and Hlavác [30]) and radial basis functions are selected as kernel argument, regularization constant, and kernel functions, respectively.

Table 3 shows the SVC results. From Table 3, we can see that the recognition rate of rolling element fault is $86 \%$ and the inner race fault is $96 \%$. For the other three cases, that is, the normal case, the outer race fault, and compound fault, we obtain high performance results with 100\% accuracy.
3.2. Gear Fault Diagnosis. The MFS-MG experimental platform for the gear fault simulation [29] is shown in Figure 9. Compared to the bearing fault simulator, the gear fault simulator has a slight difference; that is, a gearbox and a transmission belt are added. In addition, two normal bearings are installed at both the bearing holder on left side and the bearing holder on right side. During the experiment, the data are acquired by an accelerometer mounted on the top of the gearbox. The vibration signals of four cases are collected, that is, normal case, broken teeth, wears, and gear missing teeth. The spindle speed is $1764 \mathrm{rpm}$ and the end of the gearbox is free of loading, the gearbox transmission ratio is $1.5: 1$, the gear teeth is 18, pitch diameter is $28.575 \mathrm{~mm}$, and helix angle is $33^{\circ} 41^{\prime}$. Two fault cases, that is, a gear with broken teeth and a gear with missing teeth, are shown in Figures 10(a) and 10(b), respectively.

Similar to bearing fault diagnosis, we use the same sampling frequency and also 163800 data points (gear vibration signal). To detect the faults using SVC, we proceed with the same procedures as shown in Section 3.1. The original signals and the corresponding eight energy ratios distribution maps for four cases, including normal case and three fault cases (broken teeth, wear and missing teeth), are shown in Figures $11,12,13$, and 14 , respectively.

The first three training samples of the first signal and the first three test samples of the second signal are shown in Tables 4 and 5, respectively. To represent the four cases numerically, we define the standard labels, that is, the normal case, the broken teeth case, the wear case, and the missing teeth case as 1 to 4 , respectively. 
TABLE 5: Gear test samples.

\begin{tabular}{|c|c|c|c|c|c|c|c|c|}
\hline \multirow{2}{*}{ Cases } & \multicolumn{8}{|c|}{ Energy ratios in each frequency band } \\
\hline & 1 & 2 & 3 & 4 & 5 & 6 & 7 & 8 \\
\hline \multirow{3}{*}{ Normal } & 0.5055 & 0.3774 & 0.0664 & 0.0074 & 0.0285 & 0.0099 & 0.0034 & 0.0015 \\
\hline & 0.4699 & 0.3989 & 0.0656 & 0.0089 & 0.0345 & 0.0160 & 0.0048 & 0.0014 \\
\hline & 0.4881 & 0.4068 & 0.0555 & 0.0088 & 0.0257 & 0.0099 & 0.0038 & 0.0014 \\
\hline \multirow{3}{*}{ Broken teeth } & 0.5709 & 0.3092 & 0.0712 & 0.0152 & 0.0235 & 0.0068 & 0.0027 & 0.0006 \\
\hline & 0.5425 & 0.3387 & 0.0668 & 0.0163 & 0.0238 & 0.0069 & 0.0038 & 0.0012 \\
\hline & 0.5727 & 0.3254 & 0.0645 & 0.0111 & 0.0176 & 0.0059 & 0.0022 & 0.0006 \\
\hline \multirow{3}{*}{ Wear } & 0.8120 & 0.1040 & 0.0188 & 0.0055 & 0.0330 & 0.0255 & 0.0009 & 0.0003 \\
\hline & 0.8267 & 0.0975 & 0.0175 & 0.0055 & 0.0301 & 0.0215 & 0.0009 & 0.0003 \\
\hline & 0.8521 & 0.0831 & 0.0190 & 0.0061 & 0.0236 & 0.0141 & 0.0008 & 0.0010 \\
\hline \multirow{3}{*}{ Missing teeth } & 0.1902 & 0.3251 & 0.1063 & 0.0116 & 0.1712 & 0.1581 & 0.0334 & 0.0041 \\
\hline & 0.1932 & 0.3082 & 0.0844 & 0.0244 & 0.1607 & 0.1909 & 0.0299 & 0.0083 \\
\hline & 0.2200 & 0.2558 & 0.1340 & 0.0092 & 0.1816 & 0.1727 & 0.0212 & 0.0055 \\
\hline
\end{tabular}

TABLE 6: Gear recognition results.

\begin{tabular}{lcccc}
\hline Cases & Training samples & Testing samples & Classification label & Recognition rate \\
\hline Normal & 50 & 50 & 1 & $94 \%$ \\
Broken teeth & 50 & 50 & 2 & $96 \%$ \\
Wear & 50 & 50 & 3 & $100 \%$ \\
Missing teeth & 50 & 50 & 4 & $100 \%$ \\
\hline
\end{tabular}

The SVC results are shown in Table 6. The recognition rates for the normal case, the broken teeth, the wear, and the missing teeth are $94 \%, 96 \%, 100 \%$, and $100 \%$, respectively.

Based on the above experimental investigations, the proposed hybrid approach is reasonably effective for detecting different kinds of faults in both bearings and gears.

\section{Conclusion}

This paper proposes a hybrid approach using wavelet packet and SVC to classify faults for bears and gears. The collected vibration signals are directly employed as inputs without any pretreatment. The signals are decomposed by wavelet packet and the energy ratios of all frequency bands are calculated to construct the feature vectors so as to train and test the support vector machines to predict the fault type of bearings and gears. Experimental investigations for bearing fault diagnosis and gear fault diagnosis are made using MFS-MG experimental platform (the bearing fault simulator and the gear fault simulator). The results show that the proposed hybrid approach is effective to the rotating and the transmission structures. Moreover, the present approach has a good recognition rate not only for a single fault but also for the compound fault.

\section{Conflict of Interests}

The authors declare that there is no conflict of interests regarding the publication of this paper.

\section{Acknowledgments}

The authors are grateful for the support from the National Science Foundation of China (no. 51175097), the Zhejiang Provincial Natural Science Foundation for Excellent Young Scientists (no. LR13E050002), and the Project sponsored by SRF for ROCS, SEM.

\section{References}

[1] B. Samanta, "Gear fault detection using artificial neural networks and support vector machines with genetic algorithms," Mechanical Systems and Signal Processing, vol. 18, no. 3, pp. 625644, 2004.

[2] B.-S. Yang, W.-W. Hwang, D.-J. Kim, and A. C. Tan, "Condition classification of small reciprocating compressor for refrigerators using artificial neural networks and support vector machines," Mechanical Systems and Signal Processing, vol. 19, no. 2, pp. 371390, 2005.

[3] B. Samanta and K. R. Al-Balushi, "Artificial neural network based fault diagnostics of rolling element bearings using timedomain features," Mechanical Systems and Signal Processing, vol. 17, no. 2, pp. 317-328, 2003.

[4] B. Sreejith, A. K. Verma, and A. Srividya, "Fault diagnosis of rolling element bearing using time-domain features and neural networks," in Proceedings of the IEEE Region 10 Colloquium and 3rd International Conference on Industrial and Information Systems (ICIIS '08), Kharagpur, India, December 2008.

[5] O. R. Seryasat, M. Aliyari Shoorehdeli, F. Honarvar, and A. Rahmani, "Multi-fault diagnosis of ball bearing based on features extracted from time-domain and multi-class support vector machine (MSVM)," in Proceedings of the IEEE International 
Conference on Systems, Man and Cybernetics (SMC '10), pp. 4300-4303, October 2010.

[6] Z. L. Liu, Q. Z. Lu, Y. L. Wang, and C. Y. Wei, "A direct selection method of feature frequency," in Emerging Research in Artificial Intelligence and Computational Intelligence-International Conference, vol. 315, pp. 479-486, 2012.

[7] V. K. Rai and A. R. Mohanty, "Bearing fault diagnosis using FFT of intrinsic mode functions in Hilbert-Huang transform," Mechanical Systems and Signal Processing, vol. 21, no. 6, pp. 2607-2615, 2007.

[8] P. B. Tribeni, D. Swagatam, R. Joydeb, and A. Ajith, "Implementation of a new hybrid methodology for fault signal classification using short -time fourier transform and support vector machines," in Advances in Intelligent and Soft Computing, vol. 73, pp. 219-225, 2010.

[9] P. Jun, G. H. Yan, W. Xuan, T. Jing, and Y. G. Yang, "Gear fault detection with wigner-viller distribution based cepstrum approach," International Conference on Computer Engineering and Technology, vol. 1, pp. 1500-1502, 2010.

[10] P. K. Kankar, S. C. Sharma, and S. P. Harsha, "Rolling element bearing fault diagnosis using wavelet transform," Neurocomputing, vol. 74, no. 10, pp. 1638-1645, 2011.

[11] P. W. Tse, W.-X. Yang, and H. Y. Tam, "Machine fault diagnosis through an effective exact wavelet analysis," Journal of Sound and Vibration, vol. 277, no. 4-5, pp. 1005-1024, 2004.

[12] H. Li, L. Fu, and H. Zheng, "Bearing fault diagnosis based on amplitude and phase map of Hermitian wavelet transform," Journal of Mechanical Science and Technology, vol. 25, no. 11, pp. 2731-2740, 2011.

[13] P. K. Kankar, S. C. Sharma, and S. P. Harsha, "Rolling element bearing fault diagnosis using wavelet transform," Neurocomputing, vol. 74, no. 10, pp. 1638-1645, 2011.

[14] N. G. Nikolaou and I. A. Antoniadis, "Rolling element bearing fault diagnosis using wavelet packets," NDT and E International, vol. 35, no. 3, pp. 197-205, 2002.

[15] X. F. Fan and M. J. Zuo, "Gearbox fault detection using Hilbert and wavelet packet transform," Mechanical Systems and Signal Processing, vol. 20, no. 4, pp. 966-982, 2006.

[16] T. Y. Wang and J. Z. Lin, "Fault diagnosis of rolling bearings based on wavelet packet and spectral kurtosis," in Proceedings of the 4th International Conference on Intelligent Computation Technology and Automation (ICICTA '11), pp. 665-669, March 2011.

[17] D. Thukaram, H. P. Khincha, and H. P. Vijaynarasimha, "Artificial neural network and support vector machine approach for locating faults in radial distribution systems," IEEE Transactions on Power Delivery, vol. 20, no. 2 I, pp. 710-721, 2005.

[18] A. Crampton and J. C. Mason, "Detecting and approximating fault lines from randomly scattered data," Numerical Algorithms, vol. 39, no. 1-3, pp. 115-130, 2005.

[19] G. F. Bin, J. J. Gao, X. J. Li, and B. S. Dhillon, "Early fault diagnosis of rotating machinery based on wavelet packetsempirical mode decomposition feature extraction and neural network," Mechanical Systems and Signal Processing, vol. 27, no. 1, pp. 696-711, 2012.

[20] Q. Hu, Z. J. He, Z. S. Zhang, and Y. Y. Zi, "Fault diagnosis of rotating machinery based on improved wavelet package transform and SVMs ensemble," Mechanical Systems and Signal Processing, vol. 21, no. 2, pp. 688-705, 2007.

[21] G.-M. Xian and B.-Q. Zeng, "An intelligent fault diagnosis method based on wavelet packer analysis and hybrid support vector machines," Expert Systems with Applications, vol. 36, no. 10, pp. 12131-12136, 2009.

[22] C. Q. Shen, D. Wang, F. R. Kong, and P. W. Tse, "Fault diagnosis of rotating machinery based on the statistical parameters of wavelet packet paving and a generic support vector regressive classifier," Measurement, vol. 46, pp. 1551-1564, 2013.

[23] S. G. Mallat, A Wavelet Tour of Signal Processing, Elsevier Press, Burlington, Vt, USA, 3rd edition, 2009.

[24] T. Y. Wang and J. Z. Lin, "A tutorial on support vector regression," Statistics and Computing, vol. 14, no. 3, pp. 199-222, 2004.

[25] H. P. Huang and Y. H. Liu, "Fuzzy support vector machine for pattern recognition and data mining," International Journal of Fuzzy Systems, vol. 4, no. 3, pp. 826-835, 2002.

[26] Y. S. Zhu and Y. Y. Zhang, Support vector machine and its application in mechanical fault pattern recognition [Ph.D. thesis], Department of Mechanical Engineer, Xian Jiaotong University, Xi’an, China, 2003.

[27] J. Y. Yang and Y. Y. Zhang, "Application research of support vector machines in condition trend prediction of mechanical equipment," in Proceedings of the 2nd International Symposium on Neural Networks: Advances in Neural Networks (ISNN '05), vol. 3498 of Lecture Notes in Computer Science, pp. 857-864, chn, June 2005.

[28] C.-W. Hsu and C.-J. Lin, "A comparison of methods for multiclass support vector machines," IEEE Transactions on Neural Networks, vol. 13, no. 2, pp. 415-425, 2002.

[29] http://www.spectraquest.com/.

[30] V. Franc and V. Hlavác, "Statistical pattern recognition toolbox for matlab," Research Reports of CMP, Czech Technical University in Prague, 2004. 

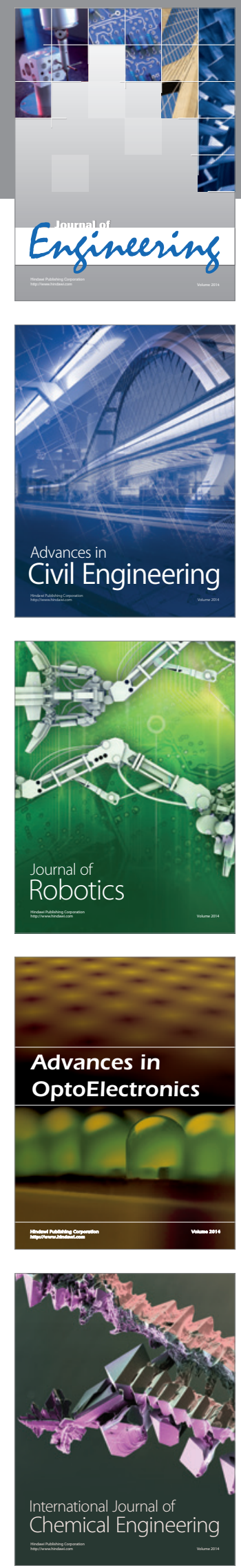

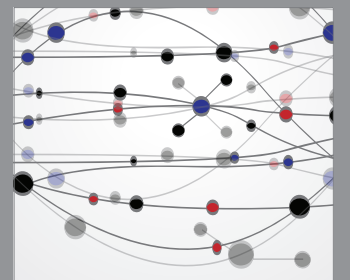

The Scientific World Journal
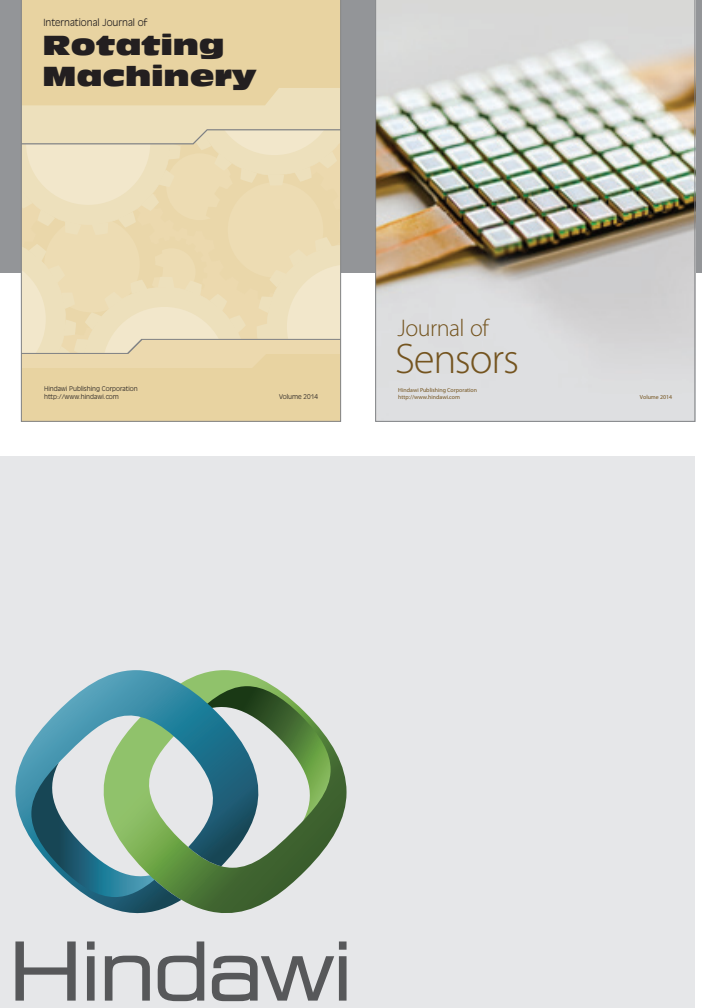

Submit your manuscripts at http://www.hindawi.com
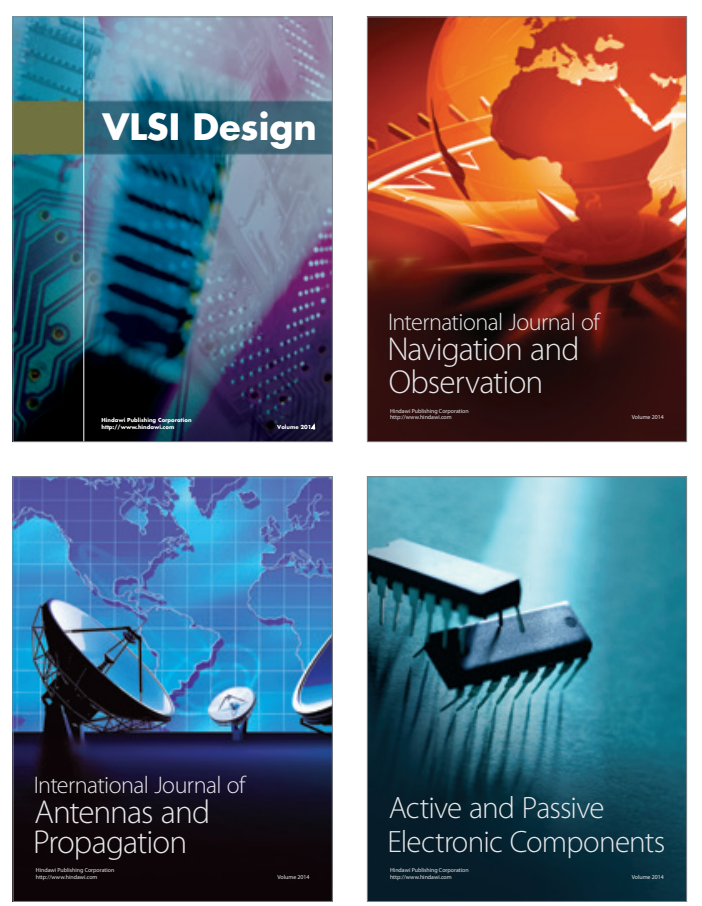
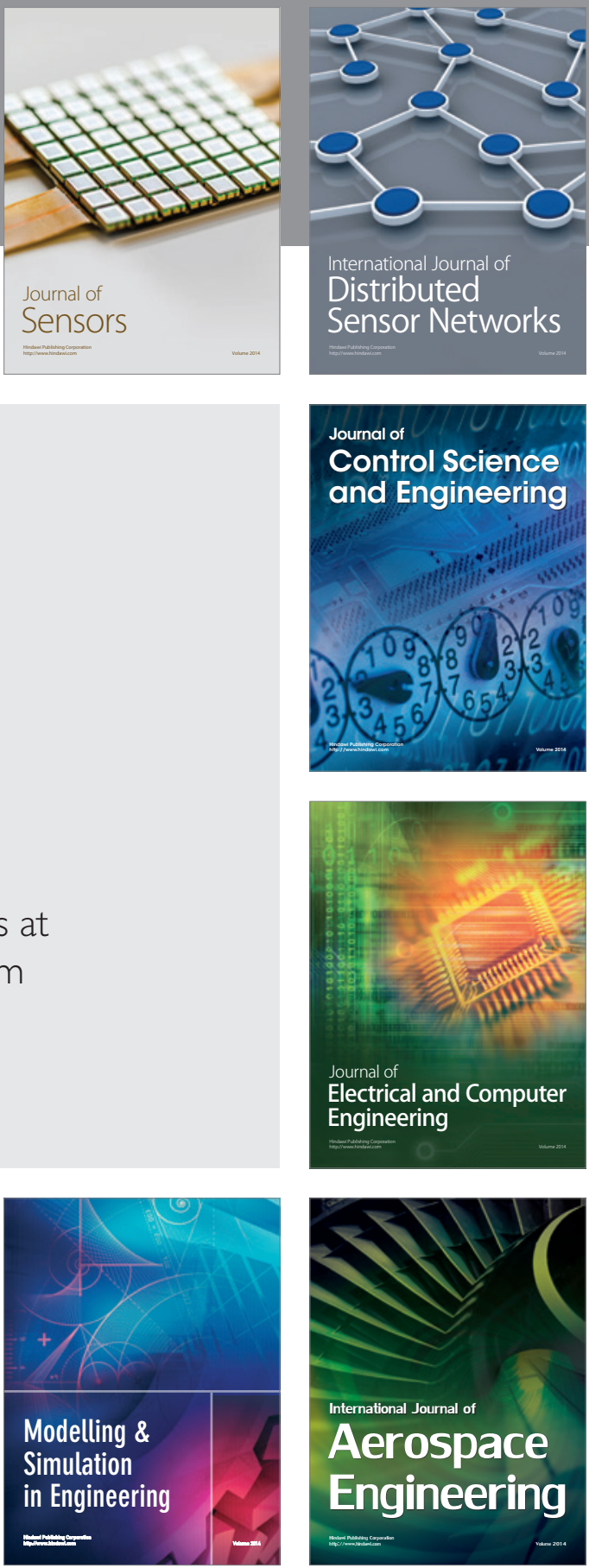

Journal of

Control Science

and Engineering
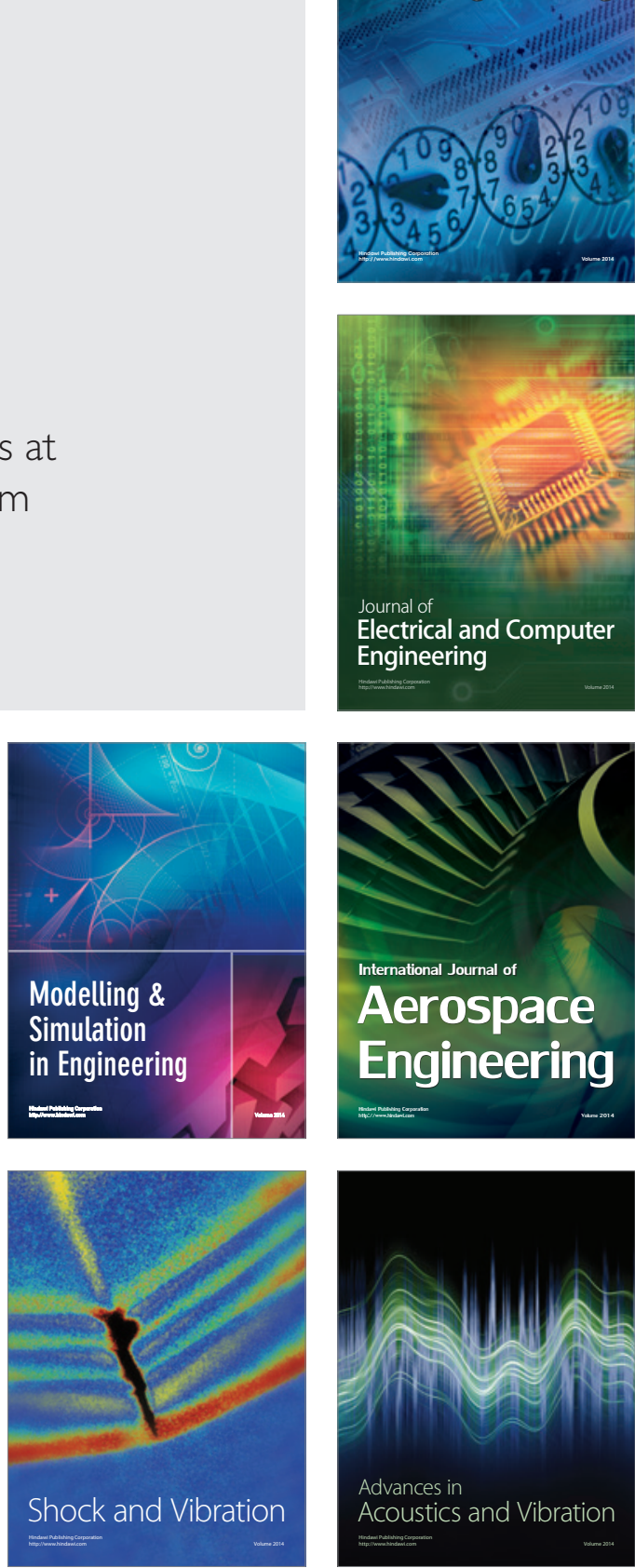